\title{
Proteomics in multiplex
}

\author{
Protein arrays and protein assays in parallel are enabling researchers to look at protein \\ interactions and activity on a large scale, as Lisa Melton finds out.
}

$\mathrm{M}$ apping the protein universe is a daunting task. Charting the interactions of proteins in the human proteome, studying their function and how their expression changes in health and disease, may take decades. Compared with about 40,000 genes in the genome, the human proteome is predicted to top 1 million. "The existence of more than a million protein isoforms in a single human being, in different cells, during his or her lifetime is not unreasonable," speculates Rolf Apweiler, Swiss-Prot coordinator at the European Bioinformatics Institute in Hinxton, UK. "These are all guesses," he cautions, but, so far, over 300 known post-translational modifications have been discovered, and these can occur $\rightarrow$ in different combinations, on different proteolytically processed forms and splice variants. A growing array of tools is allowing scientists to advance the study of the proteome, boost drug discovery and pave the way to personalized medicine.

Traditional proteomics uses techniques such as two-dimensional gel electrophoresis or chromatography, combined with mass spectrometry, to separate and identify proteins on a large scale (see Nature 424, 581-587; 2003). Tracking protein interactions and activity and determining function at this scale is an even greater task, but is beginning to be tackled by protein arrays and multiplexed assays.

To cancer researcher Julio Celis, coordinator of the Danish Centre for Translational

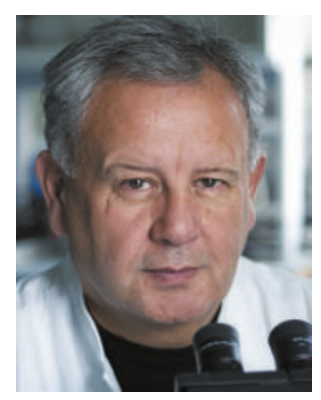

Julio Celis: keeping track of proteins. Breast Cancer Research in Copenhagen, protein arrays are a breakthrough because they allow many different proteins to be tracked simultaneously. "Discoveries have to be made using very small amounts of clinically relevant sample, and you will want to probe, say, thousands of tissues, both normal and pathological. Multiplexing allows that; it makes life a lot easier," he says.

The commonest type of protein array, or protein chip, has a large number of spots of either proteins or their ligands arranged in a predefined pattern, arrayed by robots onto coated glass slides, microplates or membranes. The array may consist of anti- bodies to bind proteins of interest (see Nature 426, 725-731; 2003), enzymes that will interact with substrates, or substrates or ligands that will interact with applied proteins.

\section{Arrays made easy}

One technical hurdle to producing a reliable array is shelf-life. Most protein arrays use antibodies as the immobilized 'probe'. "The process of depositing an antibody onto a solid surface can denature the antibody and affect its recognition properties," says Michael Hadjisavas, strategic marketing manager for Sigma-Aldrich in St Louis, Missouri. "We've developed the know-how to select the most desirable antibodies and maintain their biological activity on an array for up to two years." Sigma's off-theshelf chips provide a hassle-free introduction to protein arrays. The Panorama $\mathrm{Ab}$ microarray kit for cell signalling has 224 different antibodies, arrayed on nitrocellulose-coated glass slides. Proteins extracted from cells or tissues are labelled with the fluorescent dyes Cy3 and Cy5, and the protocol is similar to that of a DNA microarray, with the two labelled protein pools being mixed and applied to the array. The assay takes only 4-5 hours, and can be measured

\section{DO-IT-YOURSELF ARRAYS}

For those who want to spot their own arrays, a number of companies provide standard-sized $25 \times 76 \mathrm{~mm}$ coated slides especially formulated for protein arrays. Proteins arrayed on the nitrocellulose-coated FASTSlide from Schleicher and Schuell Bioscience in Cologne, Germany, are stable for up to three years at room temperature, according to the company. SuperProtein

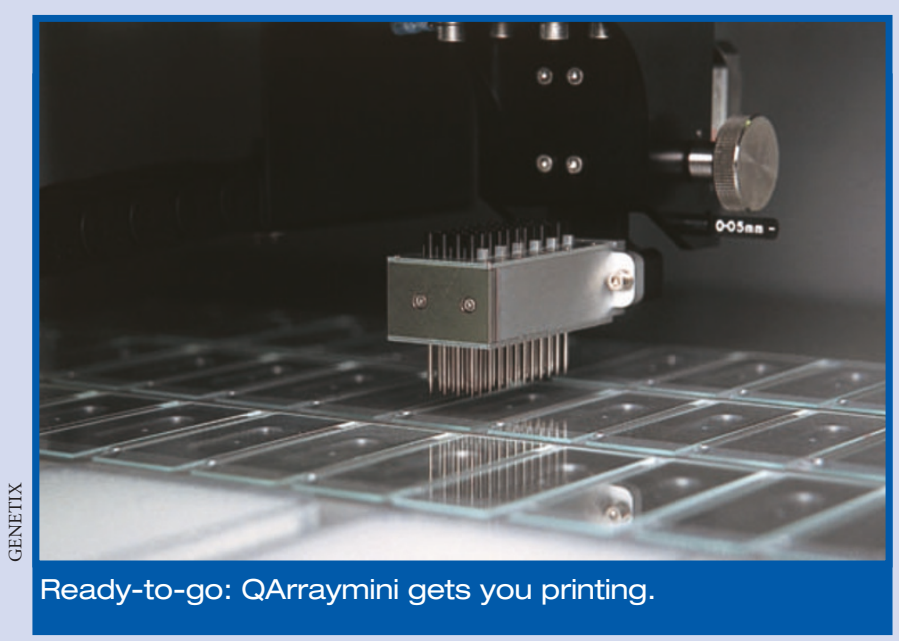

slides coated with hydrophobic polymer from TeleChem International in Sunnyvale, California, and the MaxiSorp black polymer-coated slides from Nalge Nunc in Rochester, New York, are also designed for protein arrays. $3 \mathrm{D}$ HydroGel-coated slides from PerkinElmer in Boston, Massachusetts, provide a hydrophilic environment and the capacity to hold more than a single layer of probes.

To get you started, Genetix in New Milton, UK, has the QArraymini, a ready-to go benchtop microarray spotter. "People can just get it out of the box, and get on with it," says Gail Czerepinski, product manager for Genetix. The QArraymini is available as part of a protein-array package that includes source-plate chiller, buffers, epoxy-coated slides and scanner. The spotter uses contact pin printing to array antibodies or other proteins on $25 \times 75 \mathrm{~mm}$ epoxy-coated slides, printing up to 120,000 spots on each slide. "People are printing between a few hundred and a few thousand protein spots. But they can easily scale up should they wish to," says Czerepinski. The aQuire confocal dual-laser scanner, with its own software, images Cy3 and Cy5 dyes simultaneously, with a blue laser fitted as an option.

Protein arrays made in individual wells of 96- or 384-well plastic microplates, such as the plates for the new high-throughput HTAplatform from Greiner Bio-one in Frickhausen and Scienion in Berlin, Germany, are becoming popular, as they allow economical parallel processing of many different test samples. Genetix has developed an optional module for plate arraying for their high-throughput arrayers QArray2 and QArraymax. 
using any standard fluorescence scanner.

Fluorescence is not the only detection system on offer in kit arrays. The range of chemiluminescence-based TransSignal protein-domain arrays from Panomics in Redwood City, California, is expanding, with the addition of $\mathrm{SH} 2$ and $\mathrm{WW}$ domains. The FASTQuant kit from Schleicher and Schuell in Keene, New Hampshire, combines traditional enzyme-linked immunosorbent assay (ELISA) methodology with the power of multiplexing. Antibodies to nine different human cytokines are incorporated into an array on the company's FASTSlides - nitrocellulosecoated glass slides - and 64 arrays are arranged in a microtitre plate footprint. The company plans to release six FASTQuant kits for human and mouse cytokines, angiogenesis factors and human chemokines this year.

\section{Adding content}

One bottleneck that chip manufacturers face is content. "Getting an antibody against every protein in the human proteome is a gigantic task, more so than sequencing the genome. This is not something that will happen in the next decade," says Jan van Oostrum, head of proteome sciences in functional genomics at Novartis in Basel, Switzerland.

Increasing the content is a matter of competitive advantage for Molecular Staging in New Haven, Connecticut, which uses protein chips in their work with biotech and pharma companies to identify biomarkers for diseases and help reanalyse promising drugs that, although highly effective in some patients, failed clinical trials because of unacceptable side-effects in others. "As soon as we see that a pathway is upregulated or downregulated we gain a mechanistic understanding of the disease, its progression, and patients' response to drugs," says Peter Fuller, senior vice-president of business development. "We work constantly to increase content - every four months we validate a new chip." The company's antibody-based chips use a signal-amplification technology developed at Yale University, New Haven. DNA circles attached to the detector proteins are replicated to produce a long DNA molecule that can be detected by attachment of large numbers of small detector molecules. Molecular Staging is collaborating with Eli Lilly to identify biomarkers for sepsis, a potentially deadly reaction to a blood infection that develops so rapidly that physicians find it difficult to diagnose quickly enough.

\section{Multiplexing in solution}

The challenges of constructing solid-surface arrays holding thousands of proteins with different properties are fuelling interest in protein-interaction assays in solution. Suspension-bead assays are particularly flexible, and can be adapted to both proteins and nucleic acids. The Bio-Plex system from Bio-Rad Laboratories in Hercules, California, uses Luminex's bead-based xMAP technology (see Nature 422, 917-923; 2003), as does the LiquiChip system from Qiagen Instruments in Hombrechtikon, Switzerland. For protein analysis, Bio-Plex uses antibodies from Cell Signaling Technologies in Beverly, Massachusetts, to offer ready-togo cytokine assays, and assays for phosphorylated signal transduction molecules, as well as customized arrays. Suspension-bead arrays are flexible enough to tackle any sort of protein-ligand interaction by simply coupling the required proteins or ligands to different bead populations. Luminex beads, for example, enable simultaneous quantitation of up to 100 different biomolecules in a single microplate well.

Another versatile platform is that of ACLARA Biosciences in Mountain View, California, which can support gene-expression and protein assays run side-by-side on the same sample. Detection is by proprietary eTAG reporter molecules, which are coupled to the ligands (for protein analysis these are usually antibodies), with a different eTAG for each ligand.Labelled ligands are applied to the sample in microtitre plates, or tissue sections on slides, and if the ligand binds to its target, the tag is released. Released tags are resolved, identified and quantified by capillary electrophoresis. An additional tacophoresis step concentrates the tags, taking detection sensitivity into the attomolar range. "It is possible to detect just 10 molecules," says Sharat Singh, chief technology officer at ACLARA. "In testing patient samples, we can predict whether a patient will respond or not by analysing a few analytes related to the mechanism of action of the drug and the biology of the specific target in the tumour."

The 3D HydroArray platform from Biocept in Carlsbad, California, uses a proprietary hydrogel polymer that is $95 \%$ water,

\section{A NATURAL AFFINITY}

How proteins interact with each other has become a key issue in drug discovery and development. Once a target for a drug is identified, it is also important to define how strongly it binds to other biomolecules to obtain information about selectivity. Biacore of Uppsala, Sweden, has been producing affinity-based sensor chips and instrumentation for protein-interaction analysis

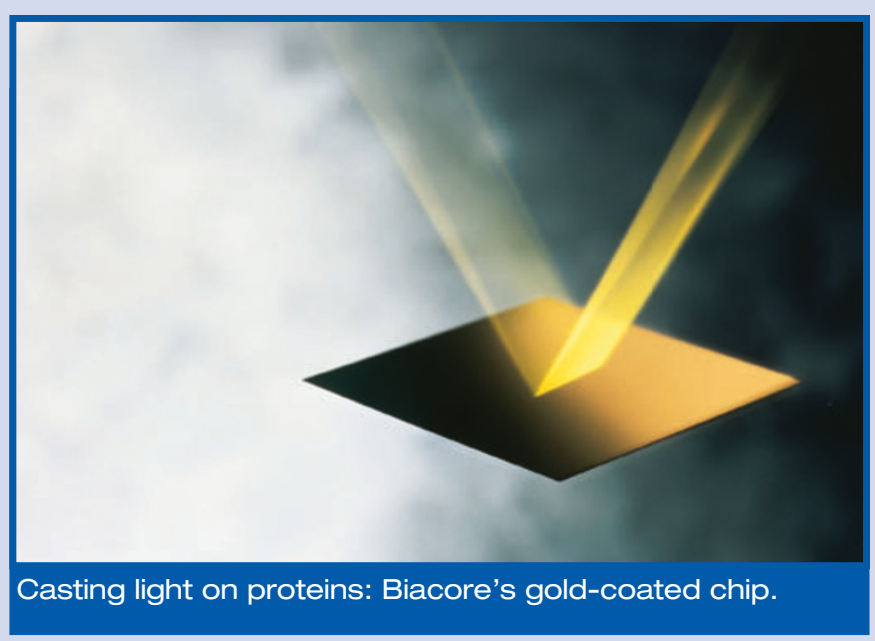

for more than a decade. The company's core technology is surface plasmon resonance (SPR), using a gold-coated glass chip onto which an array of protein-binding samples is immobilized. When the chip is illuminated, the interaction results in mass changes in the aqueous layer close to the sensor chip, that directly correlate to the refractive-index change. When molecules in the test solution bind to, or dissociate from, the immobilized protein, the refractive index rises and falls and a change is detected. Plotting the response against time allows continuous, real-time monitoring of interacting molecules. Because there is no need to label targets with fluorescent or radioactive labels, molecules can be studied in a near-native state, and the binding data closely reflect in vivo behaviour. Biacore has also developed the technology towards direct measurement of small-molecule binding to protein, for lead optimization in drug discovery, says Stephan Lofas, chief scientific officer. The company markets a variety of SPR instruments to suit different needs, from the highthroughput lab that requires unattended, fully automated runs for up to 384 samples at a time, to the smaller laboratory where researchers want to study a variety of different molecules.

The 8500 Affinity Chip Analyzer from Applied Biosystems, developed in conjunction with HTS Biosystems in Hopkinton, Massachusetts, is based on grating-coupled SPR technology. "If you are interested in target discovery, this system provides massively parallel detection of hundreds of binding events per analysis - thousands per day," says Enrico Picozza, chief technology officer at HTS Biosystems. 
enabling arrayed biomolecules (DNA or proteins) to keep their native conformation. Probe biomolecules are mixed with the prepolymer, and nanolitre droplets are arrayed on a glass slide. During curing, covalent reactions simultaneously crosslink the prepolymer, tether the biomolecules to the gel matrix and bind the droplets to the slide. The result is many levels of probes, giving high sensitivity and a broad dynamic range. Biocept has developed genomic arrays and protein arrays are coming soon.

\section{Mapping interactions}

"If you are talking proteomics, what you are looking for must not be predefined, it has to be an open approach," says van Oostrum. Reverse arrays, where the cell extract itself is spotted on a chip and probed with a large number of antibodies, is such an approach. They allow researchers to track the activation or perturbation of cellular pathways, to monitor expression of disease-related proteins, and to investigate the cellular effects of drugs, all using very small amounts of sample. Zeptosens in Witterswil, Switzerland, for example, has developed the ZeptoMark CeLyA protein-profiling system, a sensitive reverse array that uses the company's planar waveguide technology. Each chip can contain the protein content of up to 384 different cell lysates for high-throughput profiling.

"We thought that after we had identified how many genes, how many proteins, the problem would go away," says Celis. "But there are protein-protein interactions that give you different functions. Unless you

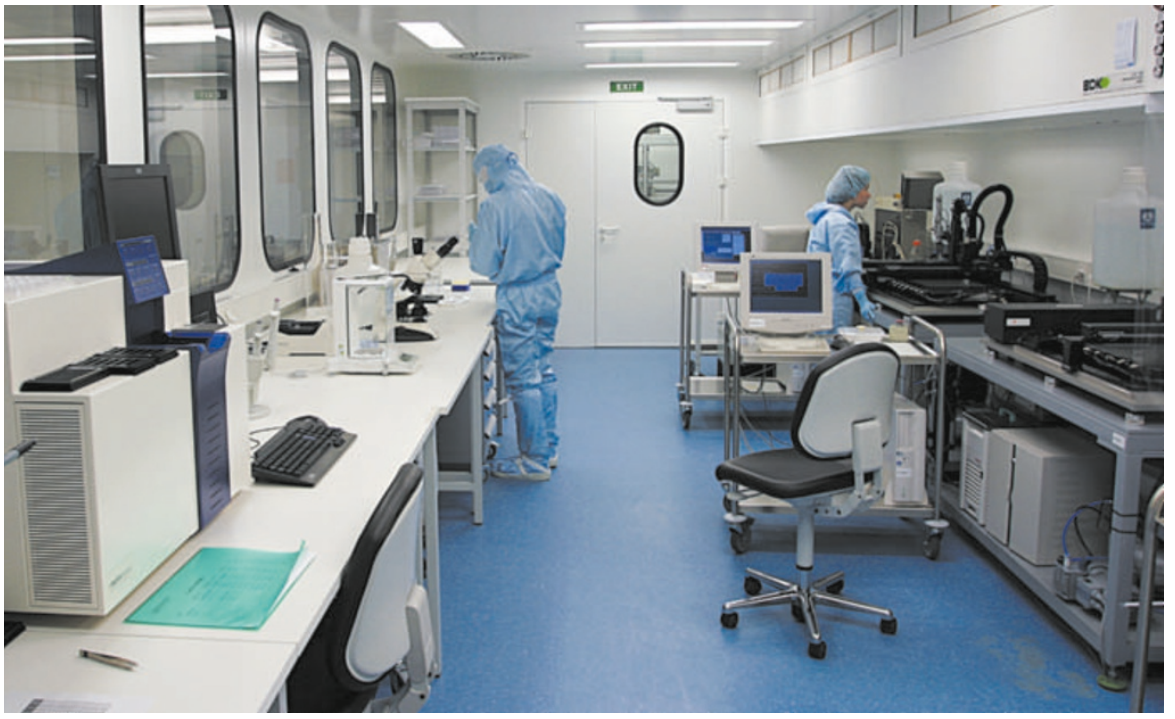

Zeptosens: microarrays must be made under rigorous clean-room conditions.

know those differences, it is difficult to come up with a drug therapy," he points out.

Giulio Superti-Furga, vice-president of biology at drug-development company Cellzome in Heidelberg, Germany, says: "We look at proteins in their own juice, with their own partners, with their own post-translational modifications." Cellzome uses two technologies based on liquid chromatography/tandem mass spectrometry to study the protein context of drugs and drug-like molecules. Potential drug compounds are immobilized and used as affinity reagents to identify binding proteins. Candidate drug targets are then 'positioned' onto cellular pathways using tandem affinity purification, originally developed by researchers at the European Molecular Biology Laboratory in Heidelberg. "You can almost derive an organizational chart, as you'd draw for a company, but for a proteome, with its drug-binding components earmarked," says Superti-Furga. Key to the approach is the compilation and visualization of data by informatics. Such maps should help identify points of intersection between different pathways, such as apoptosis and stroke for the TNF- $\alpha$ pathway and lipid metabolism for the APP pathway.

MDS Proteomics in Toronto, Canada, takes a chemiproteomics approach to iden-

\section{FAMILY BUSINESS}

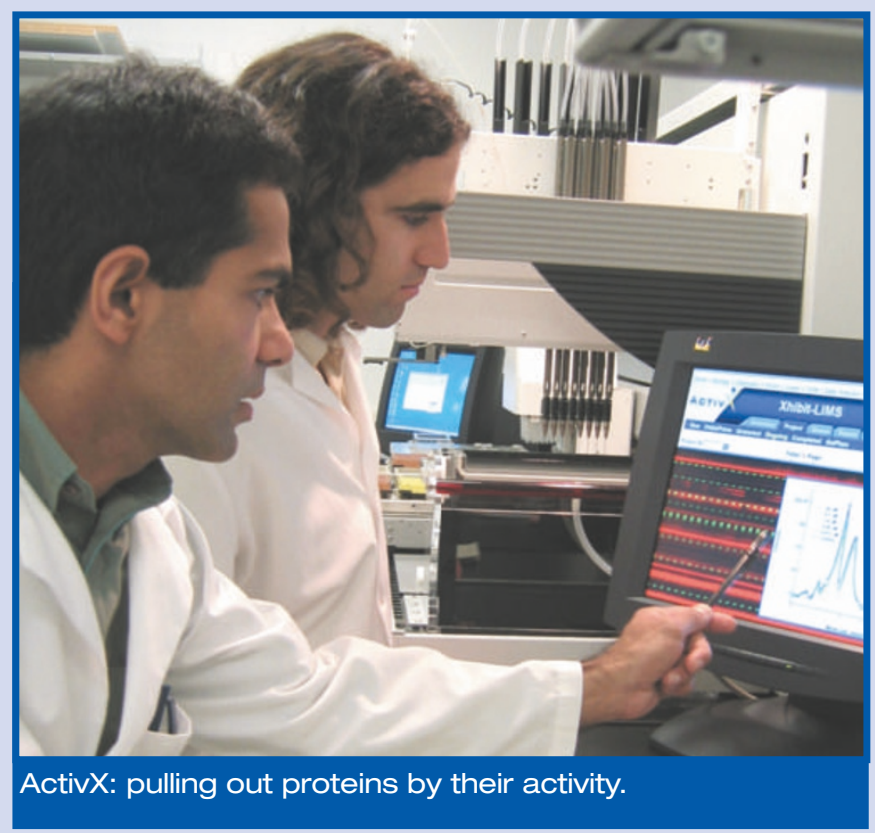

"Suppose you could develop a chemical tool so powerful that you could go into a cell and identify all the members of one family — all the protein kinases, or all the hydrolases or the phosphatases," proposes John Kozarich, president and chief scientific officer at the drug discovery and development company ActivX in La Jolla, California. The activity-based proteomics technology to do just that was originally developed by Ben Cravatt's group at the Scripps Institute and has been commercialized by ActivX.

The approach is based on a simple idea: proteins in the same family tend to have conserved active sites which a well-designed chemical reagent can pick up. In the chemistry literature are thousands of publications probing the structure and functions of proteins, defining inhibitors and ligand interactions. "We harvest that information. We are interested in tools that allow us to look at families related by a common trait," explains Kozarich.

For serine hydrolases, for example, ActivX has designed chemical tools that recognize the underlying catalytic motif - three amino acids in a particular spatial configuration. The system adds a highly fluorescent covalent tag to one of the unique amino-acid residues, and this allows all the serine hydrolases to be pulled out of a sample by capillary electrophoresis, and analysed and identified by mass spectrometry. Early in drug development, a domain scan can compare different animal models to help resolve toxicity issues. Another use is to characterize off-target interactions of drug leads, as the probes are designed to recognize just those protein motifs that are the same as the active sites that are the real drug target. 
tify primary and secondary protein targets of bioactive compounds, including those whose mechanism of action is unknown. Proteindrug complexes are pulled out of cells using a tag present on the drug. The proteins are then processed on a 'proteomics reactor', developed at the company, that simulates the physiological protein concentrations found in cells, making it more likely that the protein complexed with the drug can be chemically or biochemically processed and analysed by mass spectrometry.

A tough challenge for proteomics is the detection of integral membrane proteins which include important drug targets. By focusing on quantitative proteomic profiling of plasma-membrane proteins, drugdiscovery company Caprion in Montreal, Canada, hopes to home in on the $1 \%$ of proteins in the cell that are likely to be the most relevant drug targets. Its proprietary CellCarta platform is an integrated and automated suite of technologies for fractionation of human tissue samples and plasma, quantitative analysis and directed mass spectrometry-based identification of protein targets.

\section{Inside the intact cell}

"The big issue is, what is physiological? What is going on in vivo? How do proteins come together and interact in intact cells?" says Sam Hanash of the University of Michigan Medical School, Ann Arbor, and president of the Human Proteome Organization. This international effort in proteomics focuses on organ systems and biological fluids relevant to diseases. "Some exciting technologies are becoming available that may not be necessarily high-throughput but are more physiological." He cites methods that allow visualization of protein modifications in living cells, such as genetically encoded fluorescent indicators that detect protein-phosphorylation in signal transduction, for example.

A well-established technique for detecting protein-protein interactions is yeast two-hybrid analysis. "It's very powerful and you generate many interactions. But it is the first level of proteome mining; you then require more detailed follow-up and validation," says David Litman, senior vicepresident of R\&D and chief technology officer at BD Biosciences in San Jose, California, which makes the BD Matchmaker system for yeast two-hybrid analysis.

"A lot of proteomics is done in bulk, people taking cells and cracking them open to see what proteins are in there," says Litman. "But after scanning the whole proteome, one would want to analyse smaller sets of proteins." Antibodies to detect phosphorylated proteins are being commercialized by BD Biosiences as multiplexed immunoassays to interrogate intracellular pathways using flow cytometry. The new BD FACSArray bioanalyser accepts 96-well plates for high-throughput cellular analysis or multiplexed protein analysis using cytometric beads. A microtitre plate can be run in less than 35 minutes, detecting up to 1,000 events per well and reporting up to four fluorescent and two optical scattering parameters. With this benchtop system, researchers can study protein interactions within a given pathway without having to separate cell populations before running the assay. Reagents to detect kinases, phosphorylated proteins and other activated protein states are available, and $\mathrm{BD}$ Biosciences is developing preconfigured kits.

Protein-capture microarrays are limited as they stand, partly because of nonspecific binding by the capture agents, says James Wang, chief technology officer at Hypromatrix in Worcester, Massachusetts. "Additional protein microarray formats are needed," he says. To increase specificity and expand array applications, Hypromatrix has developed the Staining AntibodyArray to simultaneously detect and localize large numbers of cellular antigens in intact cells. Detector antibodies are arrayed on one support, while the cells are attached to a second. When the two surfaces are apposed, antibodies dissociate from the array and bind to cell-surface antigens. After the supports are separated, secondary antibodies are used to profile the antigens revealed and their cellular location.

The proteomics boom continues. And although cutting-edge proteome analysis is expensive, the growing array of proteomics tools promises to supply researchers with a method suited to every experimental need. Lisa Melton is science writer at the Novartis Foundation, London, UK.

\section{BREAKING DOWN THE PROBLEM}

A number of companies have developed peptide arrays for detecting protein interactions, such as the interaction of protein kinases with their substrates. Using high-throughput peptide synthesis, they manufacture an entire potential protein target as overlapping peptides and array them on a chip or a membrane. Pepscan Systems in Lelystad, the Netherlands, for example, offers the pre-arrayed PepChip, with 1,200 peptide substrates for protein kinase identification and assay, while New England Peptide in Gardner,

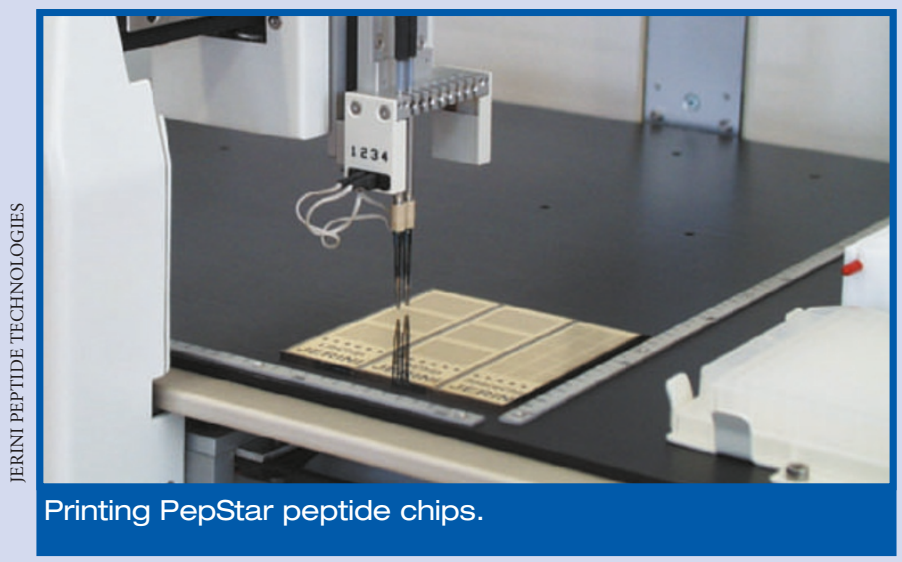

Massachusetts, has adopted the 96-well microplate format for its custom peptide arrays, with a different synthetic peptide in each well.

"The advantage is that smaller peptides are less dependent than proteins on secondary structure for their biological function," explains Holger Wenschuh of Jerini Peptide Technologies in Berlin, Germany. Jerini identifies peptide substrates for customers' orphan kinases, and for other categories of protein-modifying enzymes, such as proteases and phosphatases, using its PepStar microarray platform, in which up to 20,000 peptide sequences are synthesized on a membrane and then transferred to the complementary chip. The customer sends in their enzyme, Jerini systematically trawls the available literature and protein databases, such as Swiss-Prot and Phosphobase for potential substrates, sets up arrays, does the assay experiments and delivers the peptide substrate information, often in less than a week. Jerini also offers a ready-to-use PhosphoSite-Detector microarray kit to detect potential phosphorylation sites in kinase substrates. The readout is a phosphate-transfer event, which can be detected by autoradiography or by a phosphotyrosine-specific antibody.

Sigma's Michael Hadjisavas sees custom peptide arrays as "an area that is poised to explode". Custom peptide arrays using Sigma's PEPscreen platform are used to map epitopes, protein-protein interactions or protein-ligand interactions. "Since roughly half of all biotherapeutics are antibodies," says Hadjisavas, "it is important to demonstrate the specific peptide sequence that is being recognized in the target protein." 